\title{
THE URYSOHN-MENGER SUM FORMULA: AN EXTENSION OF THE DYDAK-WALSH THEOREM TO DIMENSION ONE
}

\author{
ALEKSANDER N. DRANIŠNIKOV and DUŠAN REPOVŠ
}

(Received 5 January 1993; revised 20 September 1993)

Communicated by H. R. Rubinstein

\begin{abstract}
Let $X$ be a finite-dimensional separable metric space, presented as a disjoint union of subsets, $X=A \cup B$. We prove the following theorem: For every prime $p, c-\operatorname{dim}_{\mathbb{Z}_{p}} X \leq \mathrm{c}-\operatorname{dim}_{\mathbb{Z}_{p}} A+\mathrm{c}-\operatorname{dim}_{\mathbb{Z}_{p}} B+1$. This improves upon some of the earlier work by Dydak and Walsh.

1991 Mathematics subject classification (Amer. Math. Soc.): primary 55M10, 55P20; secondary 54F45, 55P99.

Keywords and phrases: Urysohn-Menger sum formula, cohomological dimension, finite groups, Bockstein rings, Moore spaces, Eilenberg-MacLane complexes, Blakers-Massey theorem.
\end{abstract}

\section{Introduction}

Cohomological dimension theory (of separable metrizable spaces) is in many respects parallel to the classical (Lebesgue covering) dimension theory (see, for example, the survey by Kuz'minov [13]). This is particulary true for the cohomological dimension $c-\operatorname{dim}_{\mathbb{Z}}$ over the ring of integers $\mathbb{Z}$. The basic reason for that is the equivalence of c- $\operatorname{dim}_{\mathbb{Z}} X$ and $\operatorname{dim} X$ for finite dimensional spaces $X$, a fact which was established already in the 1930's by Aleksandrov, the founder of (co)homological dimension theory. However, in general, $c-\operatorname{dim}_{\mathbb{Z}} X$ and $\operatorname{dim} X$ need not be the same - there exist infinite dimensional spaces $X$ of finite cohomological dimension over $\mathbb{Z}$, the first such example having been found by Dranišnikov in 1987 [1, 2]. This result has had other important implications, since it provided dimension raising cell-like maps, thus solving another outstanding problem for many years in geometric (Bing) topology (see, for example, the survey by Mitchell and Repovš [14]).

The second author was supported in part by a grant from the Ministry of Science and Technology of the Republic of Slovenia.

(C) 1995 Australian Mathematical Society 0263-6115/95 \$A2.00+0.00 
One of the classical results of the Lebesgue covering dimension theory is the Urysohn-Menger sum formula [11]: it asserts that for all subsets $A$ and $B$ of $X$ such that $X=A \cup B$,

$$
\operatorname{dim} X \leq \operatorname{dim} A+\operatorname{dim} B+1 .
$$

It was only very recently that (1) was verified for the cohomological dimension over $\mathbb{Z}$ : in 1992 Rubin [15] proved that

$$
\mathrm{c}-\operatorname{dim}_{\mathbb{Z}} X \leq \mathrm{c}-\operatorname{dim}_{\mathbb{Z}} A+\mathrm{c}-\operatorname{dim}_{\mathbb{Z}} B+1 .
$$

On the other hand, it was shown in 1992 by Dranišnikov, Repovš and Ščepin [6] that the Urysohn-Menger sum formula (1) fails for cohomological dimension over arbitrary abelian groups: they have constructed subsets $A$ and $B$ of $\mathbb{R}^{4}$ such that

$$
\mathrm{c}-\operatorname{dim}_{Q / \mathbb{Z}}(A \cup B)>\mathrm{c}-\operatorname{dim}_{Q / \mathbb{Z}} A+\mathrm{c}-\operatorname{dim}_{Q / \mathbb{Z}} B+1 .
$$

(Subsequently, Dydak [9] presented a different approach to this construction.)

Rubin's argument [15] for (2) is based on the resolution method, which can be traced back to the 1970's pioneering work of Edwards (see, for example, the survey by Walsh [16]). Roughly speaking, a resolution of a polyhedron $L$ for some integer $n$, is a replacement of all $(n+1)$ and higher dimensional simplices of $L$ by the EilenbergMacLane spaces $K(\oplus \mathbb{Z}, n)$. In the 1980's Dranišnikov [1] adapted this method for the Bockstein rings $\mathbb{Z}_{p}=\mathbb{Z} / p \mathbb{Z}$ and $\mathbb{Z}_{(p)}$, that is the localization of $\mathbb{Z}$ at the prime $p$ (see also the surveys [2] and [14]). Subsequently, Dydak and Walsh [10] used this method to prove the Urysohn-Menger sum formula (1) for cohomological dimension c- $\operatorname{dim}_{R}$ over all Bockstein rings $R \in\left\{\mathbb{Z}_{(\ell)}, \mathbb{Z}_{p}\right\}_{\ell \subset \mathscr{P}, p \in \mathscr{P}}$ (where $\mathscr{P}$ is the set of all primes and $\mathbb{Z}_{(\ell)}$ is the localization of the integers at $\ell$ ), however they had to impose the restriction that both c- $\operatorname{dim}_{R} A \geq 2$ and c- $\operatorname{dim}_{R} B \geq 2$. (Note that such a restriction was unnecessary in Rubin's paper [15] since c- $\operatorname{dim}_{\mathbb{Z}} X=1$ obviously implies $\operatorname{dim} X=1$.)

Let $\operatorname{AE}(X)$ denote the class of all absolute extensors for $X$. Recall that the standard definition of the cohomological dimension of $X$ over an abelian group $G$ is

$$
\text { c- } \operatorname{dim}_{G} X \leq n \quad \text { if and only if } \quad K(G, n) \in \mathrm{AE}(X) .
$$

It follows from the work by Dranišnikov [3,4], that the Eilenberg-MacLane complexes $K(G, n)$ in the definition (4) can be replaced by the Moore spaces $M(G, n)$ :

$$
\text { c- } \operatorname{dim}_{G} X \leq n \quad \text { if and only if } \quad M(G, n) \in \mathrm{AE}(X) .
$$

Recall that $M(G, n)$ is a polyhedron such that

$$
\widetilde{H}_{i}(M(G, n)) \cong \begin{cases}G & \text { if } i=n \\ 0 & \text { if } i \neq n .\end{cases}
$$


The equivalence (5) is true for $n \geq 2$ and the conclusion c-dim $X \leq n$ also holds for $n=1$. This result allows instead for a different type of resolution of a polyhedron $L$, based on Moore spaces as building blocks.

The purpose of this paper is to remove the dimensional restrictions from the DydakWalsh theorem [10]. We were able to achieve this by using the new type of resolutions described above, that is, using the Moore spaces $M(G, n)$. We shall only give the proof for the case of the finite groups $\mathbb{Z}_{p}$ since the proof for $\mathbb{Z}_{(\ell)}$ is similar. This result was obtained in August 1992. Subsequently, Dydak [8] announced a generalization of (6) to all rings $R$ with unity.

THEOREM 1.1. For all subsets $A, B \subset X$ of a finite-dimensional separable metric space $X$ and for every prime $p$, the following holds:

$$
\mathrm{c}-\operatorname{dim}_{\mathbb{Z}_{p}}(A \cup B) \leq \mathrm{c}-\operatorname{dim}_{\mathbb{Z}_{p}} A+\mathrm{c}-\operatorname{dim}_{\mathbb{Z}_{p}} B+1 \text {. }
$$

\section{Preliminaries}

We shall require the following result from [3]:

THEOREM 2.1. Suppose $\operatorname{dim} X<\infty$ and that for some $n \geq 2$ and some abelian group $G, \mathrm{c}-\operatorname{dim}_{G} X \leq n$. Then the corresponding Moore space $M(G, n)$ is an absolute extensor for $X, M(G, n) \in A E(X)$.

We shall also need the following version of the Blakers-Massey theorem from [12, Proposition 16.30]:

Proposition 2.2. Let $(X, A) \in$ AHEP and suppose $(X, A)$ is $(n-1)$-connected and $A$ is $(s-1)$-connected. Then the homomorphism $\pi_{r}(X, A, *) \rightarrow \pi_{r}(X / A, *)$ is an $(n+s-1)$-isomorphism, for every $r>0$.

PROPOSITION 2.3. Let $p$ be any prime, $n \geq k$ and let $K$ be any $(k-1)$-connected polyhedron such that $\pi_{i}(K) \cong \bigoplus \mathbb{Z}_{p}$ for all $k \leq i \leq n$. Then there exists an inclusion $K \hookrightarrow \tilde{K}$ such that:

(i) $\pi_{i}(\tilde{K}) \cong \bigoplus \mathbb{Z}_{p}$ for $i \leq n+1$; and

(ii) the inclusion-induced homomorphism $H^{*}\left(\tilde{K} ; \mathbb{Z}_{p}\right) \rightarrow H^{*}\left(K ; \mathbb{Z}_{p}\right)$ is bijective for $* \leq k$ and surjective for $*>k$.

PROOF. Let $K^{1}=K \cup_{\varphi_{i}} B_{i}^{n+2}$ where $\left\{\varphi_{i}: \partial B_{i}^{n+2} \rightarrow K\right\}_{i \in \mathrm{N}}$ are the generators of $\pi_{n+1}(K)$. Let $\tilde{K}=\left(K^{1}, K\right)_{\mathbb{Z}_{p}}$, that is, $\tilde{K}$ is obtained from $K^{1}$ by replacing all $(n+2)$-dimensional simplices of $K^{1} \backslash K$ by the Moore spaces $M\left(\mathbb{Z}_{p}, n+1\right)$. (See [2] 
for more on this construction.) Join all copies of $M\left(\mathbb{Z}_{p}, n+1\right)$ in $\tilde{K}$ by $\operatorname{arcs}$ in $K^{1} \backslash K$ to obtain the wedge $\vee M\left(\mathbb{Z}_{p}, n+1\right)$.

Consider a couple $\left(\tilde{K}, \vee M\left(\mathbb{Z}_{p}, n+1\right)\right)$ and its exact sequence

$\cdots \bigoplus \mathbb{Z}_{p} \cong \pi_{n+1}\left(\vee M\left(\mathbb{Z}_{p}, n+1\right)\right) \stackrel{\alpha}{\rightarrow} \pi_{n+1}(\tilde{K}) \rightarrow \pi_{n+1}\left(\tilde{K}, \vee M\left(\mathbb{Z}_{p}, n+1\right)\right) \rightarrow \cdots$

By Proposition 2.2, we have that

$$
\pi_{r}(\tilde{K}, \vee M) \rightarrow \pi_{r}(\tilde{K} / \vee M) \cong \pi_{r}\left(K^{1} / \text { wedge of } \operatorname{arcs}\right) \cong \pi_{r}\left(K^{1}\right)
$$

is an isomorphism for $r=n+1$, since $\vee\left(M\left(\mathbb{Z}_{p}, n+1\right)\right)$ is $n$-connected and $(\tilde{K}, \vee M)$ is 0 -connected. By construction, $\pi_{n+1}\left(K^{1}\right)=0$; thus $\alpha$ is an epimorphism, and hence the image is $\bigoplus \mathbb{Z}_{p}$.

PROPOSITION 2.4. Let $K$ be such that $H_{k}(K)=\bigoplus \mathbb{Z}_{p}$. Then there exists an inclusion $K \hookrightarrow \tilde{K}$ such that

(i) $\pi_{i}(\tilde{K})=\bigoplus \mathbb{Z}_{p}$ for all $i$; and

(ii) $H^{i}\left(\tilde{K} ; \mathbb{Z}_{p}\right) \rightarrow H^{i}\left(K ; \mathbb{Z}_{p}\right)$ is an isomorphism for $i \leq k$ and is an epimorphism for $i>k$.

PROOF. Let $\bar{K}$ be the abelianization of $K$, that is, $\bar{K}$ is obtained from $K$ by attaching 2-cells along the commutators of all the generators of the fundamental group. Then $\pi_{i}(\bar{K})=\bigoplus \mathbb{Z}_{p}$. The map $H^{1}\left(\bar{K} ; \mathbb{Z}_{p}\right) \rightarrow H^{1}\left(K ; \mathbb{Z}_{p}\right)$ is an isomorphism because 2-disks are attached by homology trivial maps (commutators). Apply Proposition 2.3, starting from $n=1$ and $\bar{K}$ to get a sequence

$$
K \hookrightarrow K_{1} \stackrel{j_{1}}{\hookrightarrow} K_{2} \stackrel{j_{2}}{\hookrightarrow} K_{3} \hookrightarrow \cdots,
$$

where $K_{1}=\bar{K}, \pi_{i}\left(K_{n}\right)=\bigoplus \mathbb{Z}_{p}$ for $i \leq n$, and $j_{i}^{*}$ is an isomorphism in dimension one, and $j_{i}^{*}$ is an epimorphism for $*>1$. Finally, define $\tilde{K}=\underset{\lim }{\longrightarrow} K_{i}$.

PROPOSITION 2.5. If $\operatorname{dim} X<\infty$ and $\mathrm{c}-\operatorname{dim}_{\mathbb{Z}_{p}} X=1$ then $K \in \mathrm{AE}(X)$ if $\pi_{i}(K) \cong$ $\oplus \mathbb{Z}_{p}$ for all $i$.

ProOF. See $[3,7]$.

\section{3. $\left(\mathrm{cd}_{R}, n\right)$-resolutions}

DEFINITION. Let $\operatorname{cd}_{R}$ be an abbreviation for $\mathrm{c}-\operatorname{dim}_{R}$. Suppose that we have a polyhedron $K$ with some triangulation $\tau$. Then a map $\psi: \hat{K} \rightarrow K$ is called a $\left(\mathrm{cd}_{R}, n\right)$-resolution if for every simplex $\sigma \in \tau, \psi^{-1}(\sigma) \in \mathrm{AE}\left(\mathrm{cd}_{R} \leq n, \operatorname{dim}<\infty\right)$ and $\left.\psi^{-1}\right|_{K^{(n)}}: K^{(n)} \rightarrow \psi^{-1}\left(K^{(n)}\right)$ is a homeomorphism. 
Property $(*)$ of such a resolution means: For every simplex $\sigma \in \tau$ and for every integer $n \leq i$, the map $H^{i}\left(\psi^{-1}(\sigma) ; R\right) \rightarrow H^{i}\left(\psi^{-1}(\partial \sigma) ; R\right)$ is an epimorphism and $H^{n}\left(\psi^{-1}(\sigma) ; R\right) \rightarrow H^{n}\left(\psi^{-1}(\partial \sigma) ; R\right)$ is an isomorphism (for $i<n$ an isomorphism of trivial groups).

THEOREM 3.1. For all $p, n, K$, and $\tau$ there exists $a\left(\operatorname{cd}_{\mathbb{Z}_{p}}, n\right)$-resolution $\psi: \hat{K} \rightarrow K$ with the property $(*)$.

PROOF. The proof is by induction on $\operatorname{dim} K$. For $\operatorname{dim} K=n+1$, replace $(n+1)$ simplexes by $K\left(\mathbb{Z}_{p}, n\right)$ 's, by identifying the boundary of the simplex with the $n$ skeleton of $K\left(\mathbb{Z}_{p}, n\right)$. For the inductive step $m$ to $m+1$ : Suppose that $\operatorname{dim} K=$ $m+1$. Consider $\psi_{i}: \hat{K}^{i(m)} \rightarrow K^{(m)}$. Fix an $(m+1)$-simplex $\sigma$. Let $L=\psi_{i}^{-1}(\partial \sigma)$. Then by the construction $H_{n}(L) \cong \bigoplus \mathbb{Z}_{p}$. Apply Proposition 2.4 to obtain the embedding $L \hookrightarrow \hat{L}$. Then by Proposition $2.5, \hat{L} \in \mathrm{AE}\left(\operatorname{cd}_{\mathbb{Z}_{n}} \leq n, \operatorname{dim}<\infty\right)$ and so the property $(*)$ holds.

REMARK. Such a resolution with a weaker form of the property $(*)$ was constructed in [2], whereas in [10] a $\left(\mathrm{cd}_{\mathbb{Z}_{p}}, n\right)$-resolution with the property $(*)$ was constructed just for $n \geq 2$. The argument in [10] is quite different from ours and it does not allow for an extension to the case $n=1$ (hence the restrictions c-dim ${ }_{R} A \geq 2$ and c- $\operatorname{dim}_{R} B \geq 2$ in their proof of the special case of Theorem 1.1). The rest of our proof of Theorem 1.1 is to some extent similar to the argument in [10]; however, it is in many respects more elementary.

NOTATION. Let $(K, \tau)$ be a polyhedron with a triangulation $\tau$, let $f: X \rightarrow K$ be a map. Then the notation $c-\operatorname{dim}_{R}(f, \tau) \leq n$ means that the following extension problem has a solution for each subcomplex $A \subset K$ of $K$ :

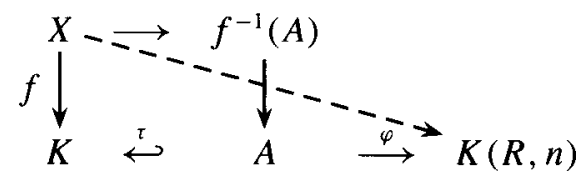

(cf. the survey of Dranišnikov [2]).

PROPOSITION 3.2. Suppose that $f: X \rightarrow K$ is a map and that for every simplex $\sigma \in \tau$ the map $H^{n}\left(f^{-1}(\sigma) ; R\right) \rightarrow H^{n}\left(f^{-1}(\partial \sigma) ; R\right)$ is an epimorphism. Then c- $\operatorname{dim}_{R}(f, \tau) \leq n$.

The proof of Proposition 3.2 is trivial. The following two theorems are taken from [2] with only minor changes: 
THEOREM 3.3. Suppose that for every open covering $\omega$ of the metric space $Y$ there exists an $\omega$-map $g: Y \rightarrow K$, with $\tau$ and there exists a $\tau$-lifting $g^{\prime}: Y \rightarrow X$ for some $f: X \rightarrow K$ with $\mathrm{c}-\operatorname{dim}_{R}(f, \tau) \leq n$. Then $\mathrm{c}-\operatorname{dim}_{R} Y \leq n$.

Here $\tau$-lifting means that if $g(x) \in \sigma \in \tau$ then $f \circ g^{\prime}(x) \in \sigma$ and the $\omega$-map has the property that $g^{-1}(\tau) \prec \omega$, where $\prec$ means refinement.

THEOREM 3.4. Suppose $\mathrm{c}-\operatorname{dim}_{R} Y \leq n$. Then for every map $g: Y \rightarrow K$ with a triangulation $\tau$, and for every $\left(\mathrm{cd}_{R}, n\right)$-resolution $\psi: \hat{K} \rightarrow K$, there is a $\tau$-lifting $g^{\prime}: Y \rightarrow \hat{K}$.

We shall also need the following assertion from [10]:

LEMMA 3.5. If $X$ is $(n-1)$-connected and $Y$ is $(m-1)$-connected then $H^{n+m+1}(X *$ $Y ; R) \cong H^{n}\left(X ; H^{m}(Y ; R)\right)$.

Proposition 3.6. Suppose that we have resolutions: $\hat{\psi}: \hat{K} \rightarrow K\left(\left(\operatorname{cd}_{R}, n\right)\right.$ resolution) and $\hat{\psi}_{1}: \hat{L} \rightarrow L\left(\left(\mathrm{~cd}_{R}, m\right)\right.$-resolution $)$ and both have the property $(*)$. Let $\hat{\sigma}=\hat{\psi}^{-1}(\sigma), \hat{\delta}=\hat{\psi}_{1}^{-1}(\delta)$. Then the following are isomorphisms:

$$
\begin{aligned}
& H^{n+m+1}(\hat{\sigma} * \hat{\delta}) \rightarrow H^{n+m+1}(\hat{\sigma} * \partial \hat{\delta}), \\
& H^{n+m+1}(\hat{\sigma} * \hat{\delta}) \rightarrow H^{n+m+1}(\widehat{\partial \sigma} * \hat{\delta}) \quad \text { and } \\
& H^{n+m+1}(\hat{\sigma} * \hat{\delta}) \rightarrow H^{n+m+1}(\widehat{\partial \sigma} * \widehat{\partial \delta})
\end{aligned}
$$

over the ring $R$, where $\widehat{\partial \delta}=\hat{\psi}_{1}^{-1}(\partial \delta)$ and $\widehat{\partial \sigma}=\hat{\psi}^{-1}(\partial \sigma)$.

PROOF. Notice that $\alpha$ is an isomorphism:

$$
\begin{array}{ccc}
H^{n+m+1}(\hat{\sigma} * \hat{\delta}) & \rightarrow & H^{n+m+1}(\hat{\sigma} * \partial \hat{\delta}) \\
\| & & \| \\
H^{n}\left(\hat{\sigma} ; H^{m}(\hat{\delta})\right) & \stackrel{\alpha}{\rightarrow} & H^{n}\left(\hat{\sigma} ; H^{m}(\partial \hat{\delta})\right) .
\end{array}
$$

Similarly the second one is isomorphism. Now the third one:

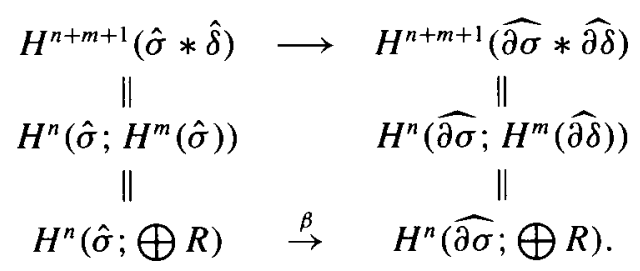

Now $\beta$ is an isomorphism, since it is for just one $R$; so it follows for finite sums $\bigoplus R$. 


\section{The proof of Theorem 1.1}

LEMMA 4.1. The map $H^{n+m+1}(\hat{\sigma} * \hat{\delta} ; R) \stackrel{\gamma}{\rightarrow} H^{n+m+1}(\partial(\hat{\sigma} * \hat{\delta}) ; R)$ is an epimorphism, where $\partial(\hat{\sigma} * \hat{\delta})=(\hat{\sigma} * \partial \hat{\delta}) \cup(\partial \sigma * \hat{\delta}) \hookrightarrow \hat{\sigma} * \hat{\delta}$.

PROOF. Consider the following Mayer-Vietoris cohomology sequences over $R$ (where $s=n+m+1$ ):

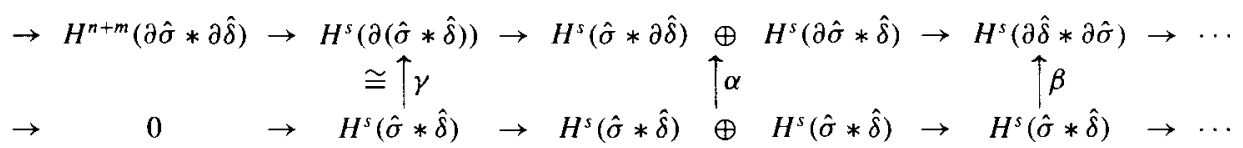

It suffices to show that $\gamma$ is an isomorphism. Suppose that $\operatorname{dim} \sigma \leq n$ and $\operatorname{dim} \delta \leq m$. Then all cohomology groups above vanish. If $\operatorname{dim} \sigma>n$ and $\operatorname{dim} \delta>m$, we have that $\partial \hat{\sigma} * \partial \hat{\delta}$ is $(n+m)$-connected, hence $H^{n+m}(\partial \hat{\sigma} * \partial \hat{\delta})=0$ and the Five Lemma yields the assertion.

It remains to consider the case when $\operatorname{dim} \sigma \leq n$ and $\operatorname{dim} \delta>m$ or vice versa. Then $\hat{\sigma} \simeq$ point: hence $\hat{\sigma} * \partial \hat{\delta} \simeq$ point, and $\hat{\sigma} * \hat{\delta} \simeq$ point. We want to show that $H^{n+m+1}(\partial(\hat{\sigma} * \hat{\delta}))=0$. It sufices to prove that $H^{n+m}(\partial \hat{\sigma} * \partial \hat{\delta})$ maps onto, since we know it maps into. To this end, consider the following diagram:

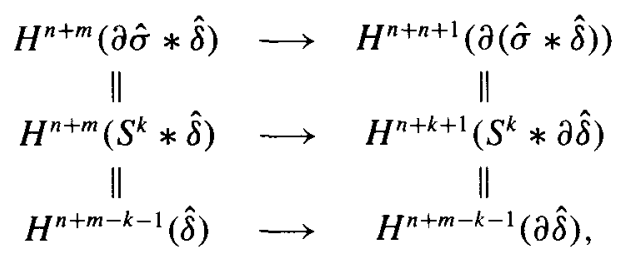

and recall that $\partial \hat{\sigma}=S^{k}$, so by the property (*) there exists an epimorphism. Hence we get a zero where we need it.

Proposition 4.2. Suppose that $\psi: \hat{K} \rightarrow K$ is a $\left(\operatorname{cd}_{R}, n\right)$-resolution and $\varphi: \hat{L} \rightarrow$ $L$ is a $\left(\mathrm{cd}_{R}, m\right)$-resolution, both with the property $(*)$. Then $\mathrm{c}-\operatorname{dim}_{R}\left(\psi * \varphi, \tau * \tau^{\prime}\right) \leq$ $n+m+1$.

ProOF. Follows by Lemma 4.1 and Proposition 3.2.

DEFINITION. Suppose that $A, B \subset X$ are disjoint subsets of $X, X=A \cup B$ and suppose that $f: A \rightarrow K$ and $g: B \rightarrow L$ are any maps. Then define the map $f \cup \cup^{*} g: A \cup B \rightarrow K * L$ as follows: Let $\bar{f}: U \rightarrow K$ be an extension of a map $f^{\prime}$, which is close to, and hence homotopic to the map $f$, over an open neighbourhood $U \subset X$ of $A$ in $X$ and let $\bar{g}: V \rightarrow L$ be an extension of a map $g^{\prime}$, which is close to, and hence homotopic to, the map $g$, over an open neighbourhood $V \subset X$ of $B$ in 
$X$ (cf. [10, Lemma 4.2]). Let $d_{A}(x)=\rho(x, X \backslash U)$ and $d_{B}(x)=\rho(x, X \backslash V)$ be the distance functions. Now, define for every $x \in X$,

$$
\left(f \cup \cup^{*} g\right)(x)=\left(f(x), g(x), d_{B}(x) /\left(d_{A}(x)+d_{B}(x)\right)\right) .
$$

Let $\pi: K * L \rightarrow[0,1]$ be the natural projection of the join $K * L$ onto the interval $[0,1]$. (Collapse $K$ and $L$ to a point, respectively.)

Lemma 4.3. Suppose that $X=A \cup B$. Then for every cover $w$ of $X$ there exist maps $\varphi_{A}: A \rightarrow K$ with a triangulation $\tau$ and $\varphi_{B}: B \rightarrow L$ with a triangulation $\tau^{\prime}$ such that $\varphi_{A} \cup \varphi_{B}$ is an $\omega$-map onto $K * L$ with respect to the triangulation $\tau * \tau^{\prime}$.

ProOF. Choose a cover $\omega_{A}$ (respectively $\omega_{B}$ ) which is a star-refinement of $\omega$ and consider the projection onto nerves, $\varphi_{A}$ (respectively $\varphi_{B}$ ).

Proposition 4.4. Suppose that $X=A \cup B$ and that there are maps $\psi: \hat{K} \rightarrow K$, $\varphi: \hat{L} \rightarrow L, f: A \rightarrow K$ with a $\tau$-lifting $f^{\prime}: A \rightarrow \hat{K}$ and $g: B \rightarrow L$ with $a$ $\tau^{\prime}$-lifting $g^{\prime}: B \rightarrow \hat{L}$. Then the map $f \cup \cup^{*} g: A \cup B \rightarrow K * L$ has $a\left(\tau * \tau^{\prime}\right)$-lifting $q: A \cup B \rightarrow \hat{K} * \hat{L}$.

ProOF. Define the lifting as follows: $q(x)=\left(f^{\prime}(x), g^{\prime}(x),\left(\pi\left(f \cup^{*} g\right)\right)(x)\right)$.

PROOF OF THEOREM 1.1. It suffices to prove Theorem 1.1 for the case when the subsets $A, B \subset X$ are disjoint; $A \cap B=\emptyset$. Indeed, if $A \cap B \neq \emptyset$ we define $B^{\prime}=B \backslash A$ and it follows that

$$
\begin{aligned}
\mathrm{c}-\operatorname{dim}_{R}(A \cup B) & =\mathrm{c}-\operatorname{dim}_{R}\left(A \cup B^{\prime}\right) \leq \mathrm{c}-\operatorname{dim}_{R} A+\mathrm{c}-\operatorname{dim}_{R} B^{\prime}+1 \\
& \leq \mathrm{c}-\operatorname{dim}_{R} A+\mathrm{c}-\operatorname{dim}_{R} B+1 .
\end{aligned}
$$

So suppose now that $A \cap B=\emptyset, A \cup B=X, \mathrm{c}-\operatorname{dim}_{R} A \leq n$ and c-dim ${ }_{R} B \leq m$. We shall prove that $\mathrm{c}-\operatorname{dim}_{R}(A \cup B) \leq n+m+1$.

To this end, consider an arbitrary cover $\omega$ of $X$ and apply Lemma 4.3 to get maps $\varphi_{A}: A \rightarrow K$ and $\varphi_{B}: B \rightarrow L$. Next, apply Theorem 3.1 to obtain the corresponding resolutions of $K$ and $L$, that is, a $\left(\operatorname{cd}_{R}, n\right)$-resolution $\psi: \hat{K} \rightarrow K$ with the property (*) and a $\left(\operatorname{cd}_{R}, m\right)$-resolution $\varphi: \hat{L} \rightarrow L$ with the property (*).

By Proposition 4.2, $\mathrm{c}-\operatorname{dim}_{R}\left(\psi * \varphi, \tau * \tau^{\prime}\right) \leq n+m+1$, and by Theorem 3.4 and Proposition 4.4, there exists a lifting $q: A \cup B \rightarrow \hat{K} * \hat{L}$ of $\varphi_{A} \cup \varphi_{B}$ which is a $\left(\tau * \tau^{\prime}\right)$-lifting. Since $\omega$ as an arbitrary covering, it follows by Theorem 3.3 that c-dim $\operatorname{dim}_{R}(A \cup B) \leq n+m+1$ as asserted. 


\section{Acknowledgements}

This paper was written during the visit by the second author to the Steklov Mathematical Institute, Russian Academy of Sciences, Moscow, in 1992 (and announced in [5]), on the basis of the agreement between the Slovenian Academy of Sciences and Arts and the Russian Academy of Sciences (1991-1995). The authors wish to acknowledge the remarks and suggestions by the referee.

\section{References}

[1] A. N. Dranišnikov, 'On a problem of P. S. Aleksandrov', Mat. Sbornik (177) 135 (177) (1988), 551-557 (in Russian). English translation Math. USSR Sbornik 63 (1988), 539-545.

[2] — , 'Homological dimension theory', Uspekhi. Mat. Nauk 43:4 (1988), 11-55 (in Russian). English translation Russian Math. Surv. 43:4 (1988), 11-63.

[3] — ' 'On intersections of compacta in Euclidean space, l', Proc. Amer. Math. Soc. 112 (1991), $267-275$

[4] - 'Extension of mappings into CW complexes', Mat. Sbornik 182 (1991), 1300-1310 (in Russian). English translation Math. USSR Sbornik 74 (1993) 47-56.

[5] A. N. Dranišnikov and D. Repovš, 'The Urysohn-Menger sum formula for cohomological dimension: An improvement of the Dydak-Walsh theorem', Abstracts Amer. Math. Soc. 14 (1993), 219, No. 93T-55-43.

[6] A. N. Dranišnikov, D. Repovš and E. V. Ščepin, 'On the failure of the Urysohn-Menger sum formula for cohomological dimension', Proc. Amer. Math. Soc. 120 (1994), 1267-1270.

[7] J. Dydak, 'Cohomological dimension and metrizable spaces', Trans. Amer. Math. Soc. 337 (1993), 219-234.

[8] _ 'Cohomological dimension and metrizable spaces, II.', Trans. Amer. Math. Soc., to appear.

[9] — 'Union theorem for cohomological dimension: A simple counterexample', Proc. Amer. Math. Soc. 121 (1994), 295-297.

[10] J. Dydak and J. J. Walsh, 'Aspects of cohomological dimension for principal ideal domains', preprint, Univ. of Tennessee, Knoxville 1992.

[11] R. Engelking, Dimension theory (North-Holland, Amsterdam, 1975).

[12] B. Gray, Homotopy theory: an introduction to algebraic topology (Academic Press, New York, 1975).

[13] V. I. Kuz'minov, 'Homological dimension theory', Uspekhi Mat. Nauk 23:5 (1968), 3-49 (in Russian). English translation Russian Math. Surveys 23:5 (1968), 1-45.

[14] W. J. R. Mitchell and D. Repovš, 'The topology of cell-like mappings', in: Proc. Conf. Diff. Geom. Topology Cala Gonone 1988, Suppl. Rend. Sem. Fac. Sci. Univ. Cagliari 58 (1988), 265-300.

[15] L. R. Rubin, 'Characterizing cohomological dimensions: The cohomological dimension of $A \cup B$ ', Topology Appl. 40 (1992), 233-263.

[16] J. J. Walsh, 'Dimension, cohomological dimension and cell-like mappings', in: Shape theory and geometric topology, Dubrovnik 1981 (eds. S. Mardešić and J. Segal), Lecture Notes in Math. 870 (Springer, Berlin, 1981) pp. 105-118. 
Department of Mathematics

Cornell University

White Hall

Ithaca, NY 14853-7901

USA

e-mail: dranish@math.cornell.edu
Institute for Mathematics Physics and Mechanics University of Ljubljana P. O. Box 64 Ljubljana 61111 Slovenia e-mail: dusan.repovs@uni-lj.si 